\title{
Molecular dynamics simulation of atomic clusters in equilibrium with a vapour
}

\author{
M. SALONEN*, I. NAPARI and H. VEHKAMÄKI \\ Department of Physical Sciences, University of Helsinki, P.O. Box 64, Helsinki FI-00014, Finland
}

(Received August 2006; in final form December 2006)

\begin{abstract}
Properties of atomic or molecular clusters surrounded by a vapour phase are needed in predicting nucleation rates and in development of phenomenological nucleation models. We have performed molecular dynamic (MD) simulations to investigate effects of thermostatting, boundary conditions and system size in cluster-vapour equilibrium. The studied system consists of Lennard-Jones (LJ) argon atoms with a potential cutoff of $6 \sigma$. We used both periodic boundary conditions and a spherical boundary with a repulsive wall to carry out the MD simulations. Interaction between the repulsive wall and vapour atoms disturbs both the density and temperature distributions of the vapour. The evolution of temperature was studied both without thermostat and with the system coupled to a Nosé-Hoover chain thermostat. We found that the particle exchange between the cluster and vapour phase is not able to equalize the temperature in constant-energy simulations. The NoséHoover thermostat performs the temperature regulation quite well. First, following an equilibration period, compact clustervapour systems were simulated. Second, to study the effects of system size, the small system is embedded into a large vapour system with the same vapour density as the original system. The equilibrium cluster size and the thermodynamical properties such as surface tension, can quite accurately be predicted by using the small system with a limited number of vapour atoms, which shortens the simulation time.
\end{abstract}

Keywords: Atomic clusters; Nucleation; Molecular dynamic simulations; Vapour

\section{Introduction}

Gas-liquid nucleation has been a focus of theoretical interest since 1920s, mostly because of its importance in atmospheric particle formation [1]. Nucleation is traditionally described in terms of so called classical nucleation theory (CNT), where the nucleating droplet is considered to consist of equilibrium bulk liquid with all the concomitant properties, such as the surface tension of planar interface. In many cases, the experimental values of nucleation rates have been far from the predictions of CNT, which has raised considerable criticism of the approximations and assumptions forming the backbone of the classical approach (see, e.g. Ref. [2] and references therein). The deficiencies of CNT are not surprising, because nucleation is a non-equilibrium process and the system consisting of vapour and cluster(s) is strongly heterogeneous.

In addition to purely theoretical attempts to improve CNT or related theories, during the last two decades computational methods have given much insight into the nucleation process. Density functional theories, Monte Carlo methods and molecular dynamics (MD) simulations have all been applied with success to give more realistic description of nucleation than CNT. Of these, MD is probably the most versatile method because it does not assume equilibrium conditions or pose any geometrical constraints on the nucleating clusters. On the downside, MD can be a very time-consuming method. The advances in computer technology have alleviated this problem greatly and in recent years nucleation simulations in very large systems have become possible for relatively simple (atoms or) molecules [3].

MD simulations of nucleation can roughly be divided into two categories [4]. In a direct simulation (e.g. Refs. $[5,6])$ a large amount of gas molecules are placed in the simulation box and the system is quenched to a supersaturated state. The simulation is then run until a critical cluster appears. Indirect simulation (e.g. Refs. $[4,7]$ ) amounts to surrounding a single cluster by a supersaturated vapour phase. The cluster grows (shrinks) if the cluster is larger (smaller) than the critical size.

*Corresponding author. Email: martta.salonen@helsinki.fi 
By performing several simulations and monitoring the molecular content of the cluster one can deduce the critical size.

The direct method is more closely related to experimental setup and it allows various stages of clusters and their properties to be measured during the simulation. However, a very long simulation time is often needed to observe a single nucleation event unless the supersaturation is much higher than in the experiments. In the indirect simulations, the critical cluster is found more quickly, but information of, for example, cluster distributions in pre-critical and post-critical stages is not gained. Critical clusters in mildly supersaturated vapours can be simulated by indirect means and since the number of molecules is much smaller than in direct simulations, systems consisting of fairly complex molecules can also be studied.

In cluster simulations, one is often interested in clusters which are in thermal equilibrium with their surroundings. Of the popular thermostatting methods used in MD simulations, we have applied the Nosé-Hoover chain thermostat $[8,9]$, which is based on coupling of the system to a set of friction parameters. Small systems coupled to a single thermostat are known to result in inadequate sampling of phase space in some cases [10,11]; NoséHoover chains, however, should fare better [8]. There are simpler thermostatting schemes, for example velocity scaling refined by Berendsen [12] and the stochastic thermostat of Andersen [13], but the Nosé-Hoover thermostat, besides giving correct canonical distribution of velocities, has the advantage of being fully timereversible and deterministic. Moreover, Nosé-Hoover thermostat is usually readily available in commercial and non-commercial MD program suites. Thermostatting of clusters with the Nosé-Hoover method has been subject to some discussion, stimulated by strange behavior observed when temperature regulation has been applied to isolated clusters. Distorted dynamics of argon clusters have been observed [14], and in bimolecular reactions the Nosé-Hoover thermostat was found incapable of exiting sufficiently the rotational modes of molecules [15]. The thermostatted system is possibly subject to other complications when the cluster is surrounded by a vapour phase, because the interaction between the parts of the system (cluster and vapour) is rather weak.

As to nucleation, different thermostatting methods can be considered equally viable if the desired properties of clusters and cluster-vapour systems are not affected by the choice of thermostat. In this paper, we study the effect of thermostatting, system size and boundary conditions on properties important in predicting nucleation rates: vapour density and pressure, cluster size and surface tension of the cluster. The first three are readily obtained from simulations. Surface tension and formation energy of a cluster are connected thermodynamically: if the formation energy is known, surface tension can be calculated [16]. Surface tension can also be found by a mechanical route using pressure tensors in spherical geometry. Pioneered by
Thompson et al. [17] in their seminal work on argon clusters, the Irving-Kirkwood definition of stress tensor [18] is commonly used for this purpose. The method was later used to find surface tension of clusters composed of Stockmayer molecules [19], water clusters [20,21] and silica nanoclusters [22]. The drawback of the pressure tensor method is the inconsistency with the thermodynamical definition of surface tension and thus it cannot be used to predict the formation energy [23].

We perform simulations thermostatted with NoséHoover chains and without any temperature control mechanism. In both the cases temperature, density and pressure distributions as well as surface tension and cluster size are evaluated and the results are then compared. Particular emphasis is placed on the boundary conditions and system size: we compare clusters embedded in small and large vapour systems and run simulations both with periodic boundary conditions and with the system enclosed in a spherical shell. In this study, we confine ourselves to simple atomistic Lennard-Jones (LJ) fluids, but the results are most likely valid for systems composed of more complicated molecules.

\section{Methods}

The simulated vapour-cluster systems are composed of atoms interacting via the $\mathrm{LJ}$ pair potential

$$
\phi(r)_{\mathrm{LJ}}=4 \epsilon\left[\left(\frac{\sigma}{r}\right)^{12}-\left(\frac{\sigma}{r}\right)^{6}\right],
$$

where $\epsilon$ and $\sigma$ are energy and length parameters and $r$ is intermolecular distance. We used the LJ parameters for argon: $\epsilon=0.24 \mathrm{kcal} / \mathrm{mol}$ and $\sigma=3.4 \AA$ and atomic mass is $40.0 \mathrm{amu}$. To speed up the calculations, we used a cutoff of $6 \sigma$ for the LJ-potential. The potential is shifted so that the potential at the cutoff distance $\mathbf{r}_{\mathrm{c}}$ is zero:

$$
\phi(r)= \begin{cases}\phi(r)_{\mathrm{LJ}}-\phi\left(r_{\mathrm{c}}\right)_{\mathrm{LJ}} & \text { for } r<r_{\mathrm{c}} \\ 0 & \text { for } r>r_{\mathrm{c}}\end{cases}
$$

Several earlier studies (e.g. Ref. [4]) show that the used cutoff distance does not induce significant errors to the cluster properties compared to those obtained with the full potential.

The MD simulations have been carried out both in a cubic box with periodic boundary conditions and in a spherical container with repulsive wall. The wall potential is the repulsive part of $\mathrm{LJ}$ potential including an additional term that shifts the potential minimum to zero:

$$
\phi(r)_{\mathrm{wall}}= \begin{cases}\phi(r)_{\mathrm{LJ}}-\phi\left(\sigma^{1 / 6}\right)_{\mathrm{LJ}} & \text { for } r<\sigma^{1 / 6} \\ 0 & \text { for } r>\sigma^{1 / 6}\end{cases}
$$

Wall potential cutoff thus lies on the distance of potential minimum. We refer to this geometry as "spherical boundary conditions" following Thompson et al. [17]. 
We started the simulation under the condition that atoms were located in a cluster with a minimum energy configuration [24]. During the equilibration a part of the atoms evaporated and formed the vapour phase. The centre-of-mass of system was adjusted to the middle of the simulation box.

A criterion is required to distinguish vapour atoms from cluster atoms. We used the Stillinger definition: an atom belongs to the cluster if it has at least one neighbour within the distance $r_{\mathrm{s}}=1.5 \sigma$. Only the largest cluster in the simulation box is regarded as the cluster, smaller entities that satisfy the Stillinger definition are considered to be part of the surrounding vapour. It has been shown that a small variation of Stillinger radius in the neighbourhood of $1.5 \sigma$ does not lead to a significant change in the cluster equilibrium size distribution [4,25].

In this study, the desired temperature was $70 \mathrm{~K}$. The temperature of the system was obtained from the kinetic energy of molecules $E_{k}$ according to the equipartition theorem

$$
T=\frac{2\left\langle E_{k}\right\rangle}{3 N k_{\mathrm{B}}} .
$$

We did no separation into rotational, translational or vibrational energies. Rotational energy of the cluster is removed at the beginning of the simulation and its contribution to the kinetic energy is insignificant throughout the simulation. The translation of the cluster is restricted due to the fixed centre of mass. The evolution of temperature was studied in microcanonical (NVE) and canonical $(N V T)$ ensembles. Since the temperature is related to the kinetic energy, the initial velocities of the atoms must be carefully adjusted to obtain equal temperatures for the cluster and the vapour, if a temperature control mechanism is not used. Temperature in NVT simulations was controlled using Nosé-Hoover thermostat with chain length of 3. A mass-like parameter, which sets the rate how quickly the system is thermostatted, was adjusted to a system with a liquid-state density: $Q_{1}=8.8 \mathrm{amu} \AA^{2} \cdot N_{\text {tot }}$ and $Q_{i}=8.8 \mathrm{amu} \AA^{2}$, $(i=2,3)$. Principally, then, we aim to regulate the temperature of the cluster.

We studied system of sizes $N_{\text {tot }}=54,138,150,250$ and 300. To study the effects of the system size, the small system was embedded into a large vapour system with the same vapour density as the original system. The total number of atoms in the larger system was 500. The additional vapour atoms were spaced randomly around the smaller simulation box with the velocities obtained from the atoms of the smaller system. This method gives several atoms the same velocity but ensures that the vapour temperature is exactly the same as in the smaller system. Our large system is comparable in size to the system simulated by Laasonen et al. [4].

The total simulation time was in all our simulations $4.8 \mathrm{~ns}$ and the time step was $6.0 \mathrm{fs}$. The equilibration in terms of cluster size and temperature took about 20,000

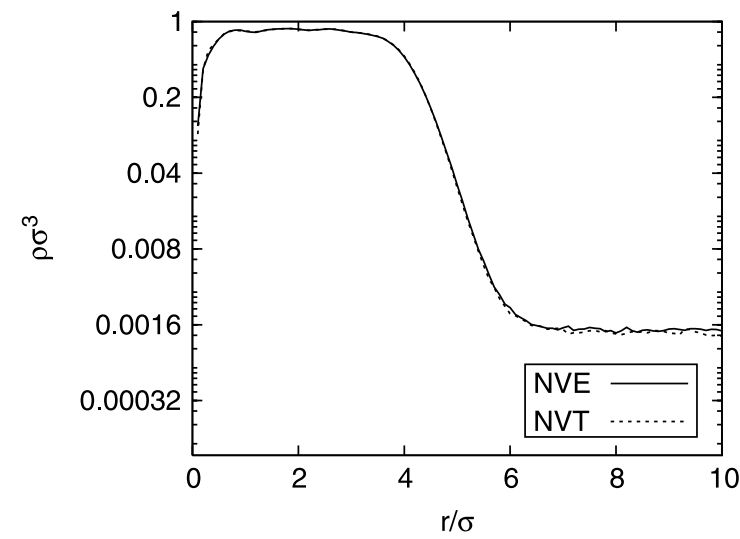

Figure 1. Radial dependence of density. Total number of atoms is 250 . A cubic simulation box with periodic boundary conditions is used.

steps and after this period the density, pressure and surface tension were calculated every 100 time steps. Density, temperature and pressure profiles were calculated by dividing the system into spherical layers of thickness $\sigma / 10$ with the origin fixed at the centre of mass of the cluster.

\section{Results and discussion}

\subsection{Density and temperature profiles}

To consider density and temperature profiles, as well as variations of the cluster size, we first used a system consisting 250 atoms as an example. Simulation boxes with periodic and spherical boundary conditions were adjusted to give a cluster size around 240 atoms. Later, we studied properties of several cluster sizes.

Examples of the radial density distributions are shown in figures 1 and 2. For a cluster simulated in cubic box, the density profiles are almost identical with and without thermostatting (figure 1). In the $N V E$-system, the velocity distribution may be different from the MaxwellBoltzmann distribution (especially in vapour phase) but

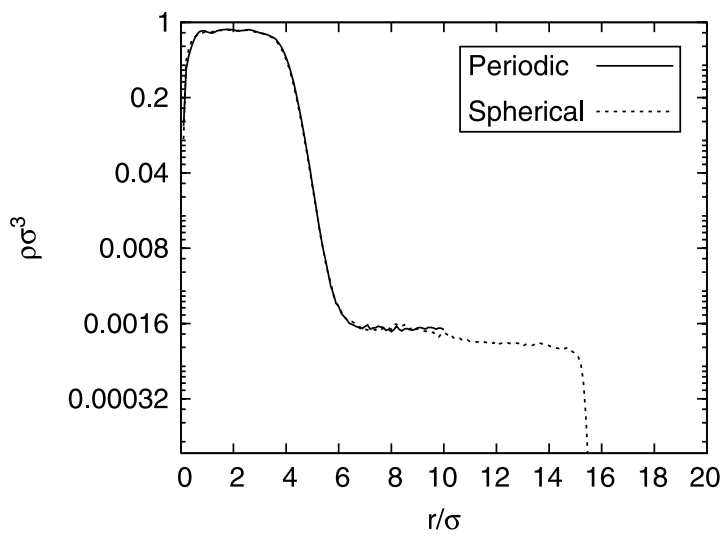

Figure 2. Radial dependence of density. Total number of atoms is 250 . Simulation in the spherical shell with spherical boundary conditions and a cubic box with periodic boundary conditions are compared. No temperature control mechanism is present. 
this does not seem to affect the density profile. In figure 2, cluster-vapour system has been simulated in cubic box with periodic boundary conditions and another simulation has been done in the spherical shell with spherical boundary conditions. No temperature control mechanism was present. Density profiles are virtually indistinguishable up to $9 \sigma$ where the vapour density in spherical shell starts to decrease due to the repulsive wall. Since repulsive interaction between atoms and wall decreases the vapour density close to the wall, to get the same average vapour density in the space unaffected by the boundary, with the same number of atoms, we have to choose a larger simulation shell if spherical boundary conditions are used. The side length of cubic box is $20 \sigma$ (volume $8000 \sigma^{3}$ ) whereas the diameter of spherical shell is $32 \sigma$ (volume $137,258 \sigma^{3}$ ). If the simulation box is chosen too large, an extensive vapour phase is formed, which lengthens the equilibration time. Interaction between the atoms of the periodic system with the images of the cluster in neighbouring cells are nonexistent because the potential cutoff distance is smaller than the distance between the cluster and its image. Small changes in starting configuration does not have an significant effect on the density distribution of the final system.

Radial dependence of the system temperature at four different simulation conditions with cubic box are shown in figure 3. In figure 3(A), the total number of atoms is 250 . Simulations are done without temperature control mechanism. Superficially, the system temperature seems rather uniform. However, we found that it is time-consuming
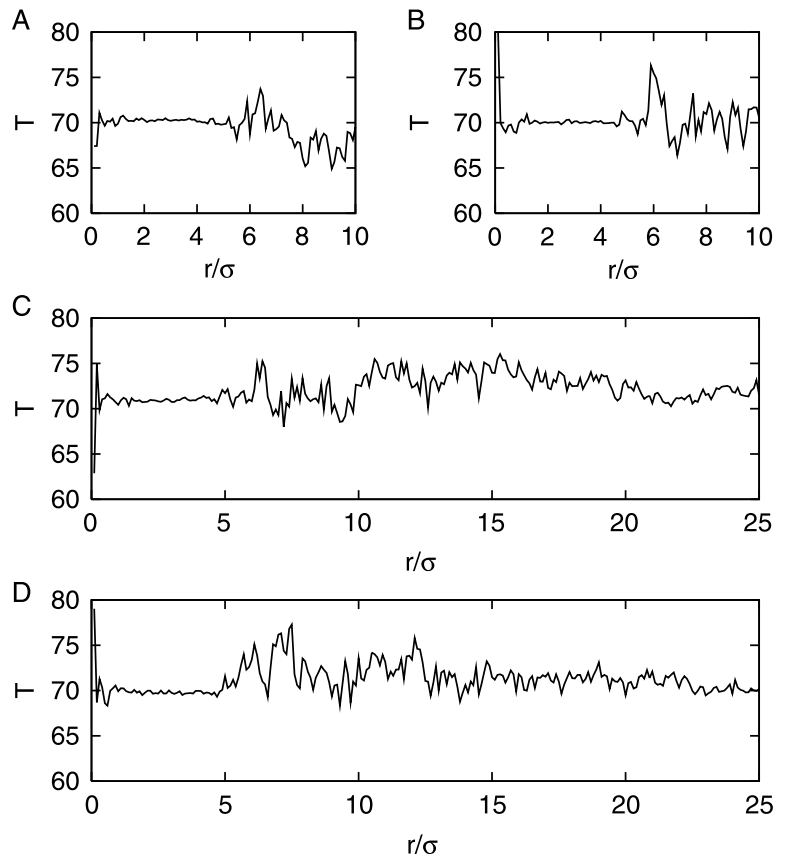

Figure 3. Radial dependence of the system temperature $T(\mathrm{~K})$. Cubic simulation box with periodic boundary conditions is used. The origin is fixed at the centre of mass of the cluster. The number of atoms in the cluster is about 240. In the figure: (A) total number of atoms $N_{\text {tot }}=250$ and no thermostat is present; (B) $N_{\text {tot }}=250$ and thermostat is used; (C) $N_{\text {tot }}=500$ and no thermostat; and (D) $N_{\text {tot }}=500$ and thermostat. to find initial parameters that lead to the correct temperature in both phases and particle exchange between cluster and vapour phase is too slow to equalize the temperatures. Similar problem were reported by Kraska [26] in constant-energy simulations of gas-liquid nucleation. System of the same size is shown figure 3(B). This time the temperature of the system is controlled by NoséHoover chain thermostat. With thermostatting, the temperature of the cluster converges fast to the desired temperature, but as the thermostat parameters were adjusted to thermostat atoms at liquid densities, the temperature control in the vapourphase was imperfect.

Figure 3(C) and (D) shows temperature profiles in enlarged systems. The clusters that are shown in figure 3(A) and (B) are embedded in an enlarged vapour phase with total number of atoms 500. Thus, the number of vapour atoms in the bigger system was about 260, whereas only about 10 atoms formed the vapour phase in the smaller system. In all figures, we can see a peak around $6 \sigma$. This indicates the fact that the atoms that escape from the cluster have higher kinetic energy than the cluster atoms on average. However, they lose their excess energy fast via the interaction with the vapour atoms further away from the cluster. The average temperatures of the cluster and vapour are in somewhat closer agreement in NVT than NVE simulations. In the vapour phase, the low density leads to poor statistics and thus the fluctuation of average temperature is several decrees. Since the average density is calculated in concentric spherical shells of constant thickness, the shell volume increases with radius, resulting better statistics near the box borders.

Temperature profiles for simulations with spherical boundary conditions are presented in figure 4 . The total number of atoms is 250 and the volume of the simulation shell is adjusted to give the same cluster size as in the cubic box with periodic boundary conditions. The cluster shown in figure $4(\mathrm{~A})$ is simulated without thermostat and
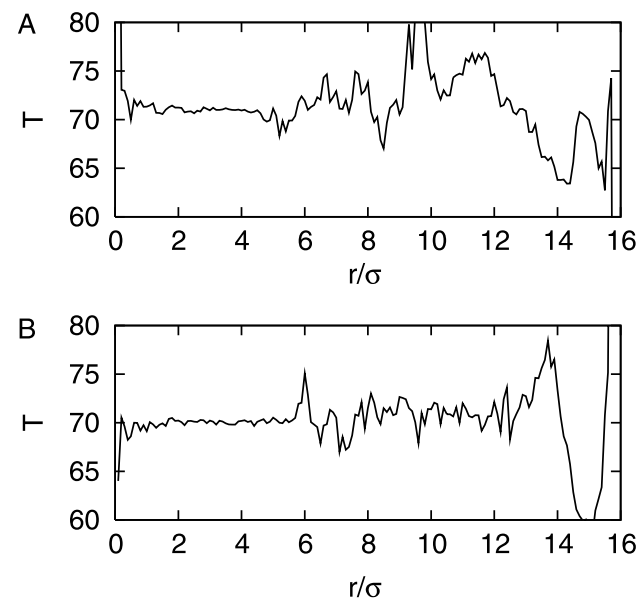

Figure 4. Radial dependence of the system temperature $T$. Simulation has been done with spherical boundary conditions. The origin is fixed to the centre of mass of the cluster. The number of atoms in the cluster is about 240; the total number of atoms is 250. In the figure: (A) no thermostat is present; and (B) Nosé-Hoover thermostat is used. 
in figure 4(B), the thermostat is used. As in the case of periodic boundary conditions, the temperature of the liquid cluster adjusts to the desired temperature, but the vapour is not so easily controlled. The repulsive wall potential has a strong effect on the temperature close to the shell boundary.

\subsection{Fluctuation of cluster size}

Once the equilibrium is reached, it is possible to study variations of the cluster size. The evolution of the cluster size is shown in figure 5. Total amount of atoms during the first $4.9 \mathrm{~ns}$ is 250 . Cubic box with periodic boundary conditions was used without thermostatting. After that system is embedded in an enlarged vapour phase and the total number of atoms are 500. As we can see from the figure, the size variation is larger when more vapour atoms are present. Due to the small amount of vapour atoms in the small system, evaporation or condensation of atoms affect the vapour density immediately and the fluctuation is subdued.

Even if we increase the system size, the average cluster size does not change. This confirms that any effects caused by the closeness of the boundary in the small system do not affect significantly the cluster-vapour equilibrium. We did some test simulations, where the small-system cluster was placed in a larger vapour environment with the vapour density increased or decreased by $30 \%$. The cluster size started immediately to chance and eventually after a couple of nanoseconds, settled to fluctuate around a new equilibrium value.

The size distribution for a cluster consisting around 240 atoms is shown in figure 6 in four different simulation conditions. The narrowest peak represents a cluster simulated with a small amount of vapour atoms and no thermostat is used. When we embed the cluster in an enlarged vapour phase, the size distribution profile became broader; due to the larger amount of vapour atoms, the cluster size can vary more than in the small system.

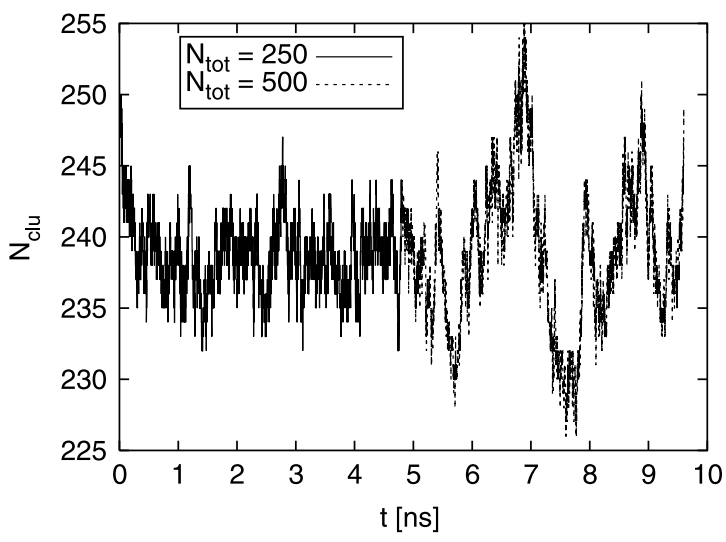

Figure 5. Temporal fluctuations of the cluster size. After $4.9 \mathrm{~ns}$, small system consisting of 250 atoms is embedded in an enlarged vapour phase and the total number of atoms is 500 . No thermostat is present and a cubic box with periodic boundary conditions are used.

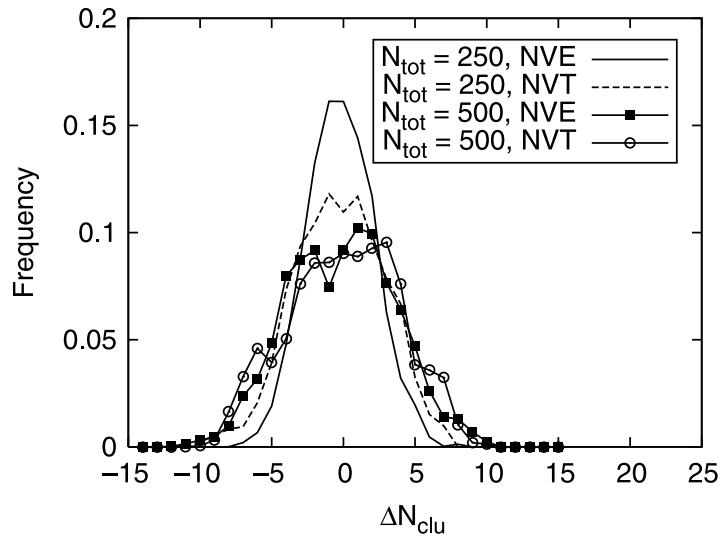

Figure 6. The variation in the cluster size in four different simulation conditions. Solid line shows cluster size distribution for a cluster simulated without thermostat in a small system $\left(N_{\text {tot }}=250\right)$ and dashed line is for a cluster with thermostat. The line with filled squares and the line with circles are size distributions in the enlarged system $\left(N_{\text {tot }}=500\right)$ simulated without and with the thermostat. A cubic box with periodic boundary conditions are used.

The size distribution is broader in a thermostatted system than in a NVE-system.

We have simulated three other cluster sizes using different total number of atoms and different sizes of simulation boxes. Vapour pressure in each system coincides with the equilibrium vapour pressure of the respective cluster. In figure 7, we compare the cluster size distribution in the small system obtained via $N V E$ simulations (solid line) with the $N V T$ simulations (dashed line), again using the cubic box. As we can see from the figure, the most probable size is almost the same with and without a thermostat. In all the systems except the largest one thermostatting causes broader cluster size distribution. The thermostat clearly alters the cluster dynamics. Asymmetrical size distribution for the smallest cluster size can be explained by the system size. Distribution should also broaden to larger sizes, but the

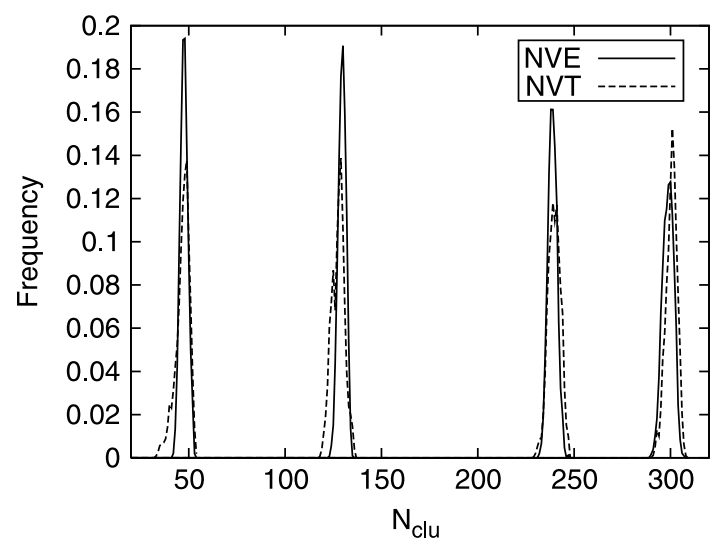

Figure 7. The cluster size distribution in the four different equilibrium system. Each cluster size has been simulated using different amount of atoms $(54,138,150,250$ and 300) and different size of simulation box (volume $1754 \sigma^{3}, 5496 \sigma^{3}, 6064 \sigma^{3}, 8000 \sigma^{3}$ and $8729 \sigma^{3}$ ). The simulations have been done without (solid line) and with (dashed line) the thermostat. A cubic box with periodic boundary conditions are used. 
vapour atoms run out when the cluster grows enough and further increase is not possible.

If a small cluster, for example $N_{\text {clu }}=40$, is embedded in enlarged vapour phase, spontaneous cluster formation occurs in the vapour because the vapour density is quite high. Similar phenomenon is not observed in a small system, where the number of vapour atoms is very small. Coagulation of the original cluster with clusters formed in the vapour distorts the equilibrium and the stabilization time is very long. After a long simulation time system settles down to another equilibrium state, e.g. bigger cluster and more rare vapour phase.

Especially in the small systems, our cluster can be considered to be in a stable equilibrium with the vapour, because the response of the cluster size to a change in the vapour density is very rapid, thus constraining the cluster to an almost fixed size. This contrasts with unstable critical clusters in an open system, where continuous growth is possible [27]. The difference, however, is of minor importance in the present study, because we do not simulate the actual formation of the clusters out of the vapour phase.

\subsection{Vapour pressure and surface tension}

We calculated the normal and transversal components of the pressure tensor as a function of distance from the centre of mass of the simulated cluster using Irwing-Kirkwood definition and the general condition of mechanical equilibrium. Calculation of the Irwing-Kirkwood pressure tensor is described in Ref. [17]. Since in a homogeneous phase the normal and transversal components of pressure tensor are equal, we are able to calculate vapour pressure: $p_{v}=p_{N}=p_{T}$. The cluster sizes as a function of vapour pressures are shown in figure 8 . We calculated the vapour pressure for all four system sizes shown in the figure 7. Both periodic and spherical boundary conditions were used and simulations were done with and without thermostat. The presented vapour pressures are estimated average pressures

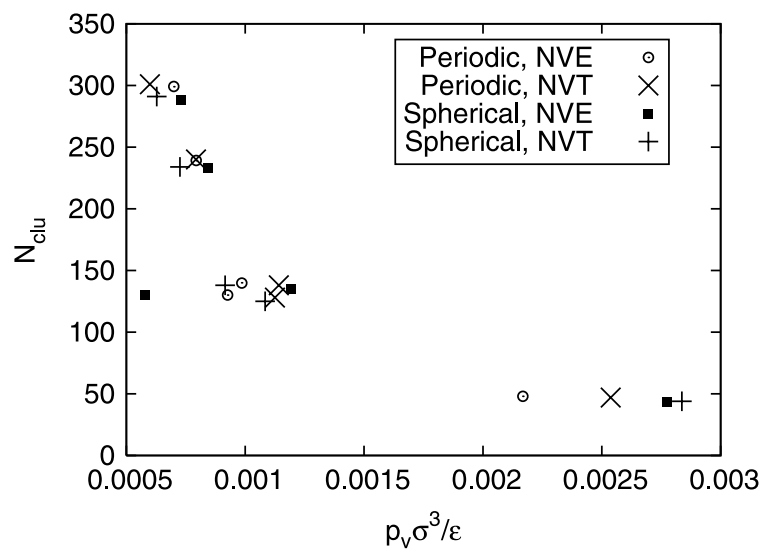

Figure 8. The cluster size as a function of the vapour phase pressure. Results are shown for four different simulation conditions. Simulations have been done with thermostat or without it and boundary conditions are either spherical or periodic.

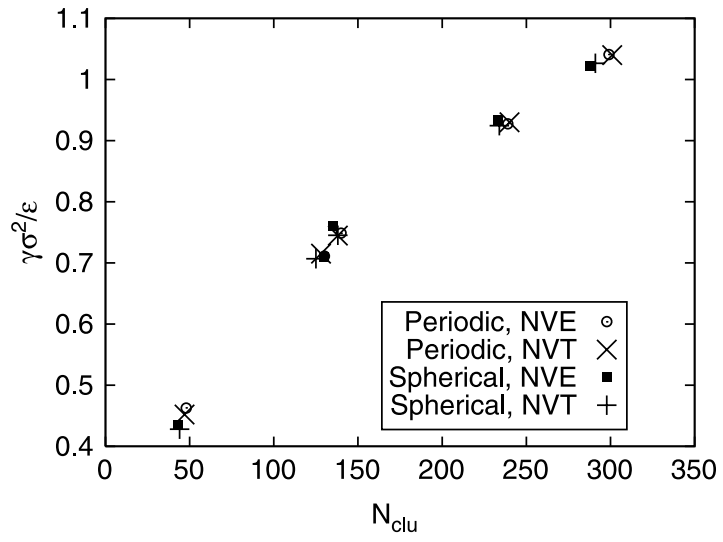

Figure 9. Surface tension as a function of the cluster size. Surface tension has been calculated for same clusters as in figure 8 .

in vapour phase sufficiently long away from both the cluster and the edge of the simulation box. Although the pressures obtained from different simulation runs deviate somewhat, no clean method-dependent trends can be seen. Due to the very low temperature of vapour phase $(56 \mathrm{~K})$, vapour pressure around a cluster consisting 130 atoms and simulated with spherical boundary conditions and without thermostat deviates from the other simulation results.

We used the mechanical route to calculate surface tension of simulated cluster,

$$
\begin{aligned}
\gamma^{3}= & -\frac{1}{8}\left(p_{l}-p_{g}\right)^{2} \\
& \times\left\{R_{\infty}^{3} p_{N}\left(R_{\infty}\right)-3 \int_{0}^{R_{\infty}} r^{2} p_{N}(r) \mathrm{d} r\right\},
\end{aligned}
$$

where $p_{l}$ is pressure in the middle the cluster, $p_{g}$ is pressure of the bulk vapour and $R_{\infty}$ is a large radius at which the normal component of pressure $p_{N}$ has the bulk vapour phase value [17].

We calculated the surface tension of clusters for the same systems as in the previous figure. Figure 9 shows the surface tension as a function of the cluster size. The surface tension increases with increasing cluster size. The estimated surface tension value of LJ fluid in bulk liquid-vapour equilibrium is $1.43 \sigma^{2} / \epsilon$ [28]. Differences in values of surface tension are not significant between the different simulation methods. This is hardly surprising, because, as we already recognize from the figures 1 and 2 , there is no visible difference in density profiles between the different simulation methods. The calculated surface tension values are in a qualitative agreement with the data by Thompson et al. [17].

\section{Conclusions}

In this paper, we compared the equilibrium properties of cluster-vapour systems in different simulation conditions. We presented density and temperature profiles, vapour pressures, surface tensions and cluster size distributions. 
Our main goal was to study effects of a temperature control mechanism on simulated cluster-vapour equilibrium. For this purpose, we applied Nosé-Hoover chain thermostat and compared the results to simulations without thermostatting. While we chose to adjust the thermostat parameters to thermostat the cluster, we found that the temperature of surrounding vapour was in good agreement with the temperature of cluster. If a thermostat was not used, it was time-consuming to find initial velocities that led to the desired temperature in both phases, because particle exchange between the cluster and the vapour phase was not able not equalize the temperatures. However, if a spatial uniformity of temperature was achieved, the cluster-vapour properties in a $N V E$ system were virtually indistinguishable from those obtained from a $N V T$ simulation. The second goal of our work was to study the effect of boundary conditions on simulated properties. Furthermore, simulations were carried out in both periodic and spherical boundary conditions. The wall-vapour interaction in the spherical shell was found to disturb both the density and temperature distributions of the vapour. The present work mainly considered small systems containing only few vapour atoms, but possible size effects was studied by embedding a small system to a larger vapour phase. Our simulations show that density distribution, surface tension and the equilibrium cluster size can quite accurately be predicted by using a small system with a very limited number of vapour atoms. Using only a small amount of vapour atoms dramatically shortens the simulation times in indirect nucleation simulations.

\section{Acknowledgements}

This work was supported in part by the Academy of Finland.

\section{References}

[1] J.H. Seinfeld, S.N. Pandis. Atmospheric Chemistry and Physics: From Air Pollution to Climate Change, Wiley, New York (1998).

[2] I.J. Ford. Statistical mechanics of nucleation: a review. J. Mech. Eng. Sci., 218, 883 (2004).

[3] T. Koishi, K. Yasuoka, T. Ebisuzaki. Large scale molecular dynamics simulation of nucleation in supercooled $\mathrm{NaCl}$. J. Chem. Phys., 119, 11298 (2003)

[4] K. Laasonen, S. Wonczak, R. Strey, A. Laaksonen. Molecular dynamics simulations of gas-liquid nucleation of Lennard-Jones fluid. J. Chem. Phys., 113, 9741 (2000).

[5] K. Yasuoka, M. Matsumoto. Molecular dynamics of homogeneous nucleation in the vapor phase. I. Lennard-Jones fluid. J. Chem. Phys., 109, 8451 (1998).
[6] S. Toxvaerd. Molecular-dynamics simulation of homogeneous nucleation in the vapor phase. J. Chem. Phys., 115, 8913 (2001)

[7] D. Zhukhovitskii. Molecular dynamics study of cluster evolution in supersaturated vapor. J. Chem. Phys., 103, 9401 (1995).

[8] G.J. Martyna, M.L. Klein, M. Tuckerman. Nosé-Hoover chains: The canonical ensemble via continuous dynamics. J. Chem. Phys., 97, 2635 (1992).

[9] M. Tuckerman, Y. Liu, G. Ciccotti, G. Martyna. Non-Hamiltonian molecular dynamics: Generalizing Hamiltonian phase space principles to non-Hamiltonian systems. J. Chem. Phys., 115, 1678 (2001).

[10] W.G. Hoover. Canonical dynamics: Equilibrium phase-space distributions. Phys. Rev. A, 31, 1695 (1985).

[11] H.A. Posch, W.G. Hoover, F.J. Vesely. Canonical dynamics of the Nosé oscillator: Stability, order, and chaos. Phys. Rev. A, 33, 4253 (1986).

[12] H.J.C. Berendsen, J.P.M. Postma, W.F. van Gunsteren, A. DiNola, J.R. Haak. Molecular dynamics with coupling to an external bath. J. Chem. Phys., 81, 3684 (1984).

[13] H.C. Andersen. Molecular dynamics simulations at constant pressure and/or temperature. J. Chem. Phys., 72, 2384 (1980).

[14] S.A. Harris, I.J. Ford. A dynamical definition of quasibound molecular clusters. J. Chem. Phys., 118, 9216 (2003).

[15] E. Kelly, M. Seth, T. Ziegler. Calculation of free energy profiles for elementary bimolecular reactions by ab initio molecular dynamics: sampling methods and thermostat considerations. J. Phys. Chem. A, 108, 2167 (2004).

[16] A. Laaksonen, I. Napari. Breakdown of the capillarity approximation in binary nucleation: a density functional study. J. Phys. Chem. B, 105, 11678 (2001)

[17] S.M. Thompson, K.E. Gubbins, J.P.R.B. Walton, R.A.R. Chantry, J.S. Rowlinson. A molecular dynamics study of liquid drops. J. Chem. Phys., 81, 530 (1984).

[18] J.H. Irving, J.G. Kirkwood. The statistical mechanical theory of transport processes. IV. The equations of hydrodynamics. J. Chem. Phys., 18, 817 (1950).

[19] A.P. Shreve, J.P.R.B. Walton, K.E. Gubbins. Liquid drops of polar molecules. J. Chem. Phys., 85, 2178 (1986).

[20] E.N. Brodskaya, J.C. Eriksson, A. Laaksonen, A.I. Rusanov. Local structure and work of formation of water clusters studied by molecular dynamics simulations. J. Colloid Interface Sci., 180, 86 (1996).

[21] V.V. Zakharov, E.N. Brodskaya, A. Laaksonen. Surface tension of water droplets: A molecular dynamics study of model and size dependencies. J. Chem. Phys., 107, 10675 (1997).

[22] I.V. Schweigert, K.E.J. Lehtinen, M.J. Carrier, M.R. Zachariah. Structure and properties of silica nanoclusters at high temperatures. Phys. Rev. B, 65, 235410 (2002).

[23] P.R. ten Wolde, D. Frenkel. Computer simulation study of gas2013liquid nucleation in a Lennard-Jones system. J. Chem. Phys., 109, 9901 (1998).

[24] D. Wales, J. Doye, A. Dullweber, M. Hodges, F. Naumkin, F. Calvo, J. Hernández-Rojas, T. Middleton. The Cambridge Cluster Database. Available online at: http://www-wales.ch.cam.ac.uk/ CCD.html (accessed 1 June 2006).

[25] B. Senger, P. Schaaf, D.S. Corti, R. Bowles, J.-C. Voegel, H. Reiss. A molecular theory of the homogeneous nucleation rate. I. Formulation and fundamental issues. J. Chem. Phys., 110, 6421 (1999).

[26] T. Kraska. Molecular-dynamics simulation of argon nucleation from supersaturated vapor in the NVE ensemble. J. Chem. Phys., 124, 54507 (2006).

[27] D. Reguera, R.K. Bowles, Y. Djikaev, H. Reiss. Phase transitions in systems small enough to be clusters. J. Chem. Phys., 118, 340 (2003).

[28] M. Mecke, J. Winkelmann, J. Fischer. Molecular dynamics simulation of the liquid-vapor interface: The Lennard-Jones fluid. J. Chem. Phys., 107, 9264 (1997). 